\title{
Article \\ Clinical Utility and Limitation of Diagnostic Ability for Different Degrees of Dysplasia of Intraductal Papillary Mucinous Neoplasms of the Pancreas Using ${ }^{18}$ F-Fluorodeoxyglucose-Positron Emission Tomography/Computed Tomography
}

\author{
Yuto Hozaka ${ }^{1}{ }^{1}$, Hiroshi Kurahara ${ }^{1}$, Hideyuki Oi ${ }^{1}$, Tetsuya Idichi ${ }^{1}$, Yoichi Yamasaki ${ }^{1}$, Yota Kawasaki ${ }^{1}$, \\ Kiyonori Tanoue ${ }^{1}$, Megumi Jinguji ${ }^{2}$, Masatoyo Nakajo ${ }^{2}$, Atsushi Tani ${ }^{2}$, Akihiro Nakajo ${ }^{1}$, Yuko Mataki ${ }^{1}(\mathbb{D}$, \\ Yoshihiko Fukukura ${ }^{2}$, Hirotsugu Noguchi ${ }^{3}$, Michiyo Higashi ${ }^{3}$, Takashi Yoshiura ${ }^{2} \mathbb{D}$, Akihide Tanimoto ${ }^{3}$ and \\ Takao Ohtsuka ${ }^{1, *}$
}

check for updates

Citation: Hozaka, Y.; Kurahara, $\mathrm{H}_{\text {. }}$ Oi, H.; Idichi, T.; Yamasaki, Y.;

Kawasaki, Y.; Tanoue, K.; Jinguji, M.; Nakajo, M.; Tani, A.; et al. Clinical Utility and Limitation of Diagnostic Ability for Different Degrees of Dysplasia of Intraductal Papillary Mucinous Neoplasms of the Pancreas Using ${ }^{18}$ F-FluorodeoxyglucosePositron Emission Tomography/ Computed Tomography. Cancers 2021, 13, 4633. https://doi.org/10.3390/ cancers 13184633

Academic Editor: Atsushi Masamune

Received: 2 August 2021

Accepted: 14 September 2021

Published: 15 September 2021

Publisher's Note: MDPI stays neutral with regard to jurisdictional claims in published maps and institutional affiliations.

Copyright: (C) 2021 by the authors. Licensee MDPI, Basel, Switzerland. This article is an open access article distributed under the terms and conditions of the Creative Commons Attribution (CC BY) license (https:// creativecommons.org/licenses/by/ $4.0 /)$.
1 Department of Digestive Surgery, Breast and Thyroid Surgery, Graduate School of Medical and Dental Sciences, Kagoshima University, 8-35-1 Sakuragaoka, Kagoshima City 890-8520, Japan; k6958371@kadai.jp (Y.H.); h-krhr@m3.kufm.kagoshima-u.ac.jp (H.K.); k6207619@kadai.jp (H.O.); k3352693@kadai.jp (T.I.); k3825645@kadai.jp (Y.Y.); k5968102@kadai.jp (Y.K.); wilson@m.kufm.kagoshima-u.ac.jp (K.T.); anakajo@m.kufm.kagoshima-u.ac.jp (A.N.); mataki@m.kufm.kagoshima-u.ac.jp (Y.M.)

2 Department of Radiology, Graduate School of Medical and Dental Sciences, Kagoshima University, 8-35-1 Sakuragaoka, Kagoshima City 890-8520, Japan; megu@m.kufm.kagoshima-u.ac.jp (M.J.); masatoyo@m3.kufm.kagoshima-u.ac.jp (M.N.); at-tani@m.kufm.kagoshima-u.ac.jp (A.T.); fukukura@m.kufm.kagoshima-u.ac.jp (Y.F.); yoshiura@m3.kufm.kagoshima-u.ac.jp (T.Y.)

3 Department of Pathology, Graduate School of Medical and Dental Sciences, Kagoshima University, 8-35-1 Sakuragaoka, Kagoshima City 890-8520, Japan; h-noguchi@kufm.kagoshima-u.ac.jp (H.N.); east@m2.kufm.kagoshima-u.ac.jp (M.H.); akit09@m3.kufm.kagoshima-u.ac.jp (A.T.)

* Correspondence: takao-o@kufm.kagoshima-u.ac.jp; Tel.: +81-99-275-5361; Fax: +81-99-265-7426

Simple Summary: Elucidating risk factors for different degrees of dysplasia of intraductal papillary mucinous neoplasms (IPMNs) of the pancreas is important in determining strategies for management. In this study, we searched for risk factors for different degrees of dysplasia of IPMNs. Our study indicated that there were no useful factors that significantly differentiated low-grade dysplasia and high-grade dysplasia; however, ${ }^{18} \mathrm{~F}$-fluorodeoxyglucose-positron emission tomography / computed tomography is useful for differentiating between non-invasive and invasive IPMNs. Our results offer critical information that may determine surgical treatment strategies.

Abstract: The diagnostic value of ${ }^{18}$ F-fluorodeoxyglucose (FDG) uptake in the management of intraductal papillary mucinous neoplasms (IPMNs) of the pancreas remains unclear. This study aimed to assess the role of FDG uptake in the diagnosis of different degrees of dysplasia of IPMNs. We retrospectively analyzed the following three points in 84 patients with IPMNs: (1) risk factors to predict high-grade dysplasia (HGD) and invasive carcinoma (INV); (2) the relationship between FDG uptake and glucose transporter 1 (GLUT-1) expression; and (3) the relationship between FDG uptake and the presence of mural nodules. The histopathological diagnosis was low-grade dysplasia (LGD) in 43 patients, HGD in 16, and INV in 25. The maximum standardized uptake value (SUV-max) was significantly higher in INV than in LGD/HGD $(p<0.0001, p=0.0136)$. The sensitivity and specificity to discriminate INV from LGD/HGD were $80.0 \%$ and $86.2 \%$, respectively, using the receiver operator characteristic curve, when the optimal cutoff score of SUV-max was set at 4.03. Those values were not different between HGD and LGD. More than half of HGD patients had low GLUT-1 expression. Taken together, FDG-PET/CT is useful in distinguishing between non-invasive and invasive IPMN. Our results offer critical information that may determine surgical treatment strategies. 
Keywords: IPMN; FDG; PET; mural nodule; high-grade dysplasia; glucose transporter 1; GLUT-1

\section{Introduction}

Intraductal papillary mucinous neoplasms (IPMNs) of the pancreas originate from the pancreatic duct epithelium, and are characterized by papillary growth, pancreatic duct dilation, and mucin hypersecretion [1,2]. It has been well established that IPMNs represent one of the premalignant lesions of pancreatic carcinoma [1,3]. IPMNs have widespread pathogenicity, ranging from low-grade dysplasia (LGD) to high-grade dysplasia (HGD) and invasive carcinoma (INV), with poor prognosis, similar to pancreatic ductal adenocarcinoma (PDAC) [3,4]. Regarding treatment, IPMNs should be resected oncologically according to the stage of premalignant lesions; however, pancreatectomy has a high perioperative morbidity rate, even at high-volume centers and, therefore, resection is not recommended for all cases [5-10]. Malignancy diagnosis plays an important role in the decision of follow-up or resection. The indicators for malignant IPMNs of the pancreas were recommended in the international consensus guidelines (ICGs) known as the Sendai Guidelines (GLs), published by the International Association of Pancreatology in 2006 [4]. The disadvantages of the Sendai criteria include a large number of benign, branched-type IPMNs (BD-IPMN) in the surgical indications, resulting in a lower positive predictive value (PPV) for malignancy. Thus, the revised ICGs in 2012 (known as the Fukuoka GLs) incorporated a clinical management algorithm for BD-IPMN based on two grouped risk factors for malignant IPMN: "high-risk stigmata (HRS)", and "worrisome feature (WF)". With this revision, management of BD-IPMN changed from an aggressive to a conservative approach [11]. In clinical practice, this management algorithm using HRS and WF is useful not only for BD-IPMN, but also for both the main duct-type IPMN (MD-IPMN) and the mixed-type IPMN. In addition, the ICGs were revised in 2017, with some minor changes to HRS and WF [12]. However, these indicators inherit the limitation of a low PPV, requiring further improvement. Additionally, we occasionally need more information about the degree of dysplasia of the IPMN, depending on the patient's age, general condition, tumor location, etc. In particular, during surgical decision making, it is important to determine whether the lesion is a non-invasive or invasive IPMN.

From the perspective of malignancy diagnosis, ${ }^{18} \mathrm{~F}$-fluorodeoxyglucose (FDG)-positron emission tomography/computed tomography (PET/CT) is widely performed for diagnosis, staging, and therapeutic effect in various carcinomas [13-17]. FDG-PET is a modality that evaluates cell activity by observing FDG uptake via glucose metabolism. Neoplastic cells with activated glucose uptake and FDG uptake also increase in numbers and, therefore, can be used for evaluation [18]. In previous studies, it has been reported that FDG-PET/CT has excellent malignancy diagnostic ability in IPMN [19-21]. However, few previous studies have analyzed results according to the differing degrees of dysplasia [22-25]. In addition, it remains unclear how the abnormal accumulation of FDG is related to mural nodules presumed to contain an abundance of neoplastic cells. Therefore, in the present study, we investigated (1) the diagnostic ability of FDG-PET/CT, which separated IPMNs associated with INV and HGD, respectively; (2) the relationship between FDG uptake and the expression of glucose transporter 1 (GLUT-1), which is one of the glucose transporters involved in the glucose metabolism of IPMNs; and (3) the relationship between FDG uptake and IPMNs with mural nodules.

\section{Materials and Methods}

\subsection{Study Design and Patient Population}

A total of 100 patients underwent pancreatic resection for IPMN at the Department of Digestive Surgery, Breast and Thyroid Surgery, Kagoshima University, between 2006 and March 2021. After excluding patients (1) who did not undergo preoperative FDG-PET, (2) who underwent recombined section of concomitant PDAC distant from IPMN, and 
(3) with high blood glucose ( $>150 \mathrm{mg} / \mathrm{dL}$ ) before FDG injection, 84 patients were enrolled (Figure 1). We retrospectively reviewed the preoperative imaging data, including computed tomography (CT), magnetic resonance imaging (MRI), endoscopic ultrasonography (EUS), FDG-PET/CT, laboratory data, and clinicopathological data of the patients. For clinicopathological data, we evaluated age, sex, symptoms, tumor location, morphological subtype of IPMN, cystic size, mural nodule height, and histopathological diagnosis. Medical records and imaging data for patients who underwent surgery prior to 2017 were also retrospectively evaluated in accordance with the revised Fukuoka GLs. This study was approved by the Ethics Review Committee of Kagoshima University (approval no. 200277, date of approval: 19 March 2021). Written informed consent was obtained from all enrolled patients.

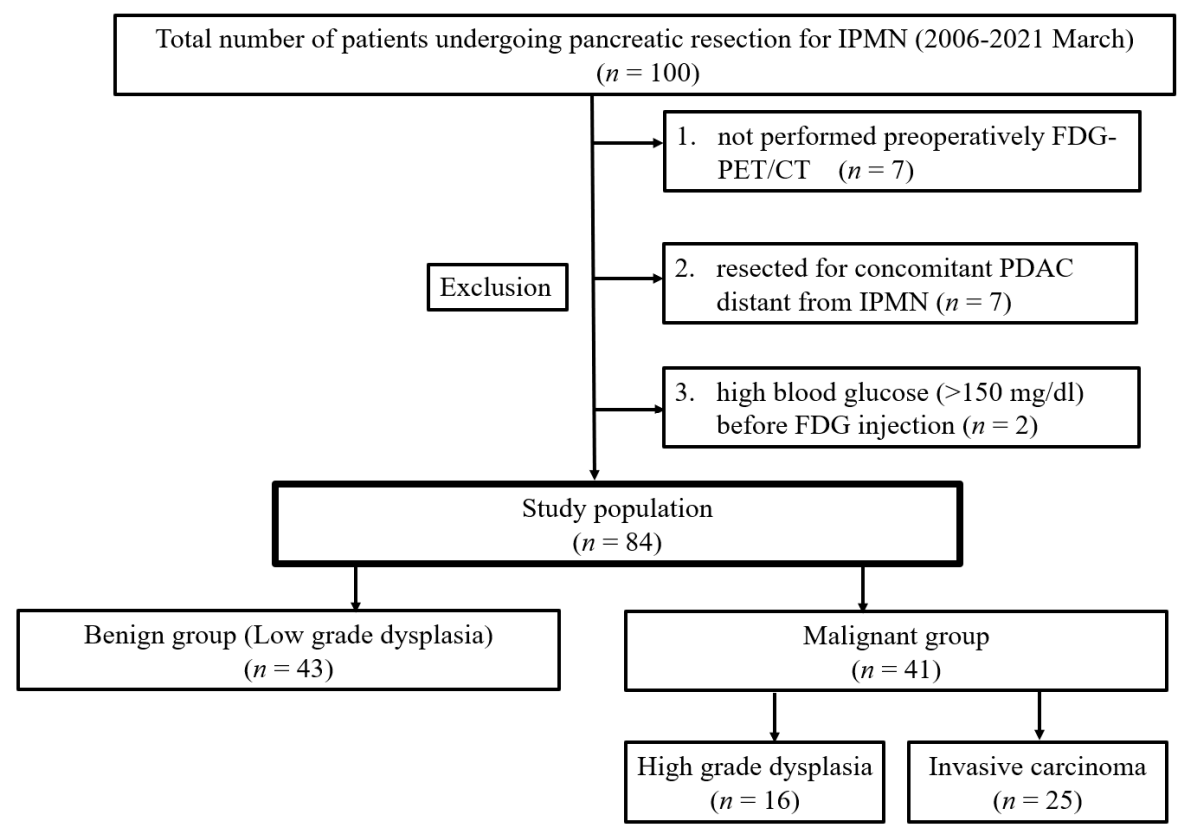

Figure 1. Distribution of the patients with IPMN. IPMN: intraductal papillary mucinous neoplasm; FDG: ${ }^{18} \mathrm{~F}$-fluorodeoxyglucose.

\subsection{Surgical Indications}

Surgical resection was determined according to the criteria of the GLs of each period, according to the Sendai, Fukuoka, and revised Fukuoka GLs.

\subsection{Imaging Modalities Protocol}

We performed contrast-enhanced CT, followed by MRI and EUS, in all patients during the initial assessment of IPMNs. Then, PET/CT was conducted in patients who were intended to undergo surgical resection. All patients underwent whole-body FDG-PET before treatment using Discovery STE (GE Medical Systems, Milwaukee, WI, USA) devices. Patients fasted for $5 \mathrm{~h}$ before receiving an intravenous administration of FDG, and FDGPET images were acquired $120 \mathrm{~min}$ after the administration of FDG. We evaluated the IPMN area with maximum FDG uptake, using contrast-enhanced CT, MRI, and EUS findings as a guide. SUV was automatically calculated as the activity concentration $\left({ }^{18} \mathrm{~F}\right.$ FDG uptake divided by the injected dose of ${ }^{18} \mathrm{~F}-\mathrm{FDG}$ (dose/g body weight)). SUV-max equals the organic radioactivity ((MB q/g)/ ${ }^{18} \mathrm{~F}-\mathrm{FDG}(\mathrm{MBq} / \mathrm{g}$ body weight)), and was calculated in the IPMN region with the highest FDG uptake. For example, the value was highest in the mural nodule in some IPMNs, but in the cyst wall or septa in others. The FDG uptake defined positives as SUV levels of 2.0 or higher. Other imaging modalitiesincluding contrast-enhanced CT, MRI, and EUS-were performed during the three months before surgery. 


\subsection{Evaluation of Mural Nodule Height}

We measured mural nodule height using contrast-enhanced CT.

\subsection{Histopathological Diagnosis}

According to the World Health Organization classification revised in 2019, the specimens were classified as LGD; HGD, including carcinoma in situ; or INV based on the retrospective pathological findings. LGD was defined as benign, whereas HGD and INV were defined as malignant. The histopathological diagnoses were confirmed by at least two pathologists.

\subsection{Immunohistochemistry (IHC) and Evaluation of IHC}

Resected specimens from 35 IPMN patients (comprising 15 HGD, 10 LGD, and 10 INV patients) were used for IHC analysis. Briefly, paraffin-embedded sections, including the area of the tumor with the highest degree of dysplasia, were sliced at a thickness of $3 \mu \mathrm{m}$. After deparaffinization and dehydration, the sections were heated at $121^{\circ} \mathrm{C}$ for $10 \mathrm{~min}$ for antigen retrieval. Sections were soaked in phosphate-buffered saline (PBS) prior to IHC analysis. The sections were also soaked in $0.3 \% \mathrm{H}_{2} \mathrm{O}_{2}$ for 10 min to block endogenous tissue peroxidase, which was followed by treatment with bovine serum for $30 \mathrm{~min}$ to reduce nonspecific binding. The sections were incubated with primary mouse monoclonal anti-GLUT-1 (dilution, 1:100; Cat. No sc-377228; Santa Cruz, TX, USA) overnight at $4{ }^{\circ} \mathrm{C}$. Sections were rinsed in PBS and visualized via standard techniques for labeled avidinbiotin immunoperoxidase staining. GLUT-1 was subsequently visualized using a 3,3'diaminobenzidine $(\mathrm{DAB})$ substrate kit. The slides were counterstained with hematoxylin. Red blood cells were used as positive controls for the intensity of GLUT-1 expression. The method of evaluating IHC for GLUT-1 expression was the same as in the previous study [26]. GLUT-1-positive expression was defined as detectable immunoreaction in cell membrane regions of $>10 \%$ of the atypical cells.

\subsection{Statistical Analysis}

Values are expressed as median and range. Differences between the two groups were analyzed using Mann-Whitney U tests for continuous variables. Multiple comparisons were performed using the Steel-Dwass tests. Fisher's exact probability tests were performed for categorized variables. The relationships between possible predictive factors and malignancy of IPMN were determined using a multivariate logistic regression model. The optimal cutoff score for discriminating differences between two groups was determined by constructing receiver operating characteristic (ROC) curves, based on the sensitivities and specificities at several predetermined cutoff points. Correlations were identified using Spearman's correlation coefficients. Any $p$-values of $<0.05$ were considered statistically significant. All statistical analyses were performed using JMP Pro 15 (SAS Institute Inc., Cary, NC, USA) and GraphPad Prism 7 (GraphPad Software, La Jolla, CA, USA).

\section{Results}

\subsection{Clinicopathological Characteristics of the Patients with IPMN}

The patients' background characteristics are summarized in Table 1. IPMN types were MD-IPMN $(n=9 ; 10.7 \%)$, BD-IPMN $(n=33 ; 39.3 \%)$, and mixed-IPMN $(n=42 ; 50.0 \%)$. The locations were the pancreatic head $(n=49 ; 58.3 \%)$, distal pancreas (pancreatic body and tail) $(n=23 ; 27.4 \%)$, and multifocal $(n=12 ; 14.3 \%)$. The pathological diagnosis was LGD $(n=43 ; 51.2 \%)$, HGD $(n=16 ; 19.0 \%)$, or INV $(n=25 ; 29.8 \%)$. No patient had a mucous mass that was initially judged as a mural nodule. 
Table 1. Patient background characteristics $(n=84)$.

\begin{tabular}{lc}
\hline \multicolumn{1}{c}{ Characteristic } & Value \\
\hline Age, y, median (range) & $70(35-87)$ \\
Sex, male, $n(\%)$ & $56(66.6)$ \\
Symptoms, presence, $n(\%)$ & $28(33.3)$ \\
History of pancreatitis, presence, $n(\%)$ & $8(9.5)$ \\
Family history of pancreatic cancer, $n(\%)$ & $8(9.5)$ \\
Coexistence of diabetes mellitus & $23(27.4)$ \\
\hline Morphological subtype, $n(\%)$ & \\
\hline Main duct type & $9(10.7)$ \\
Branch type & $33(39.3)$ \\
Mixed type & $42(50.0)$ \\
\hline Location of IPMN, $n(\%)$ & \\
\hline Head (including uncus) & $49(58.3)$ \\
Distal (left from SMV) & $23(27.4)$ \\
Multifocal & $12(14.3)$ \\
\hline Histopathological diagnosis, $n(\%)$ & \\
\hline Low grade dysplasia & $43(51.2)$ \\
High grade dysplasia & $16(19.0)$ \\
Invasive carcinoma & $25(29.8)$ \\
\hline \hline
\end{tabular}

IPMN: Intraductal papillary mucinous neoplasm; SMV: superior mesenteric vein.

The comparison between the benign and malignant groups revealed that the following clinicopathological findings were significantly more frequent in patients in malignant group compared with the benign group: male $(p=0.0128)$, presence of symptoms $(p=0.0006)$, high levels of carcinoembryonic antigen (CEA) $(p=0.0438)$, high levels of carbohydrate antigen 19-9 (CA19-9) $(p=0.0074)$, high levels of SUV-max $(p<0.001)$, and positivity for HRS (one or more). Similarly, the main pancreatic duct (MPD) size $(p=0.0484)$ and enhancing mural nodule height $(p<0.001)$ were significantly greater in the malignant group compared with those in the benign group (Table 2). In contrast, there were no significant differences between the two groups in terms of age distribution, coexistence of diabetes mellitus, concomitant pancreatitis, family history of pancreatic carcinoma, high levels of pancreatic amylase (P-AMY), morphological subtype, cystic size, cyst growth rate ( $\geq 5 \mathrm{~mm} / 2$ years), abrupt change in caliber of pancreatic duct with distal pancreatic atrophy, thickened/enhancing cyst walls, lymphadenopathy, or positivity for WF(s) (one or more).

Next, we analyzed the clinicopathological and imaging findings of the HGD, INV, and LGD groups. The rate of symptoms, CA19-9 levels, and P-AMY levels were significantly higher in the INV group than in the LGD group, but not in the HGD group. Enhancing mural nodule height and SUV-max were significantly higher in the INV group than in the LGD and HGD groups (Table 3). In contrast, when the MPD size was $\geq 10 \mathrm{~mm}$, a significant difference was observed only in the HGD group, and no difference in the INV group. Representative MRI, EUS, and PET/CT images for IPMN with LGD, HGD, and INV are shown in Figure S1.

\subsection{Malignancy Predictive Ability of ${ }^{18}$ F-Fluorodeoxyglucose-PET/CT and Mural Nodule Height for Malignant IPMN}

The SUV-max in malignant IPMNs was significantly higher than that in the LGD group $(p<0.0001)$ (Figure 2A). Among malignant IPMNs, high SUV-max was significantly more frequent in INV patients than in HGD patients $(p=0.0136)$, but there was no difference in SUV-max between the LGD and HGD groups $(p=0.4921)$ (Figure 2B). 
Table 2. Clinicopathological features between the benign and malignant groups.

\begin{tabular}{|c|c|c|c|}
\hline Characteristic & Benign $*(n=43)$ & Malignant $^{+}(n=41)$ & $p$-Value \\
\hline \multicolumn{4}{|l|}{ Clinical factor } \\
\hline Age, year, median (range) & $70(44-80)$ & $70(35-87)$ & 0.4439 \\
\hline Sex, male, $n(\%)$ & $34(79.0)$ & $22(53.6)$ & 0.0128 \\
\hline Symptoms, presence, $n(\%)$ & $7(16.2)$ & $21(51.2)$ & 0.0006 \\
\hline Coexistence of diabetes mellitus, $n(\%)$ & $8(18.6)$ & $15(36.6)$ & 0.0633 \\
\hline Concomitant pancreatitis, $n(\%)$ & $4(9.3)$ & $4(9.8)$ & 0.9449 \\
\hline Family history of pancreatic cancer, $n(\%)$ & $4(9.3)$ & $3(7.3)$ & 0.7416 \\
\hline Serum CEA, high $(\geq 3.2 \mathrm{IU} / \mathrm{L})$ & $10(23.2)$ & $18(43.9)$ & 0.0438 \\
\hline Serum CA 19-9, high ( $\geq 37$ IU/L) & $2(4.6)$ & $10(24.3)$ & 0.0074 \\
\hline Serum P-AMY, high ( $\geq 50 \mathrm{IU} / \mathrm{L})$ & $11(25.5)$ & $8(19.5)$ & 0.5055 \\
\hline \multicolumn{4}{|l|}{ Imaging factor } \\
\hline Morphological subtype, (MD/BD/mixed), $n$ & $1 / 16 / 24$ & $8 / 15 / 18$ & 0.0559 \\
\hline Main pancreatic duct size, mean \pm SD & $6.51 \pm 3.90$ & $8.63 \pm 5.74$ & 0.0484 \\
\hline Main pancreatic duct size, $(\geq 10 \mathrm{~mm}), n(\%)$ & $7(16.3)$ & $17(41.4)$ & 0.0210 \\
\hline Cystic size, mean $\pm \mathrm{SD}$ & $34.7 \pm 14.1$ & $39.8 \pm 23.6$ & 0.2194 \\
\hline Enhancing mural nodule height $\ddagger,(\geq 5 \mathrm{~mm}), n$ (\%) & $12(27.9)$ & $29(70.7)$ & $<0.0001$ \\
\hline Cyst growth rate $\geq 5 \mathrm{~mm} / 2$ years, $n(\%)$ & $3(7.0)$ & $2(4.9)$ & 0.6833 \\
\hline Abrupt change in caliber of pancreatic duct with distal pancreatic atrophy, $n(\%)$ & $1(2.3)$ & $1(2.4)$ & 0.9728 \\
\hline Thickened/enhancing cyst walls, $n(\%)$ & $12(27.9)$ & $2(4.9)$ & 0.0164 \\
\hline Lymphadenopathy, $n(\%)$ & $1(2.3)$ & $1(2.3)$ & 0.9728 \\
\hline FDG uptake, positive, $n(\%)$ & $16(37.2)$ & $28(68.2)$ & 0.0040 \\
\hline SUV-max, mean $\pm \mathrm{SD}$ & $1.95 \pm 1.39$ & $5.96 \pm 5.60$ & $<0.0001$ \\
\hline \multicolumn{4}{|l|}{ Other indicators } \\
\hline High-risk stigmata, positive, $n(\%)$ & $17(39.5)$ & $34(82.9)$ & $<0.0001$ \\
\hline Worrisome feature, positive, $n(\%)$ & $41(95.3)$ & $40(97.6)$ & 0.5811 \\
\hline
\end{tabular}

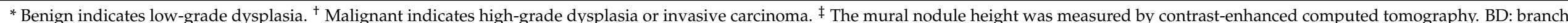

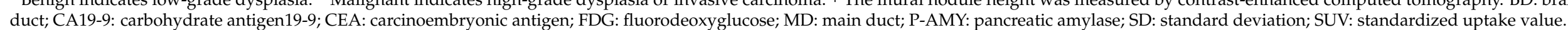


Table 3. Clinicopathological features between low-grade dysplasia, high-grade dysplasia, and invasive carcinoma.

\begin{tabular}{|c|c|c|c|c|c|c|}
\hline Characteristic & $\begin{array}{c}\text { LGD } \\
(n=43)\end{array}$ & $\begin{array}{l}\text { HGD } \\
(n=16)\end{array}$ & $\begin{array}{c}\text { INV } \\
(n=25)\end{array}$ & $\begin{array}{c}p \text {-Value (LGD vs. } \\
\text { HGD) }\end{array}$ & $\begin{array}{l}p \text {-Value (LGD vs. } \\
\text { INV) }\end{array}$ & $\begin{array}{c}p \text {-Value (HGD vs } \\
\text { INV) }\end{array}$ \\
\hline \multicolumn{7}{|l|}{ Clinical factor } \\
\hline Age, median (range), y & $70(44-80)$ & $70(49-86)$ & $70(35-87)$ & 0.5400 & 0.9126 & 0.7659 \\
\hline Sex, male, $n(\%)$ & $34(79.0)$ & $9(56.3)$ & $13(52.0)$ & 0.1951 & 0.0552 & 0.9668 \\
\hline Symptoms, presence, $n(\%)$ & $7(16.2)$ & $7(43.8)$ & $14(56.0)$ & 0.0755 & 0.0021 & 0.7393 \\
\hline Coexistence of diabetes mellitus & $8(18.6)$ & $7(43.8)$ & $8(32.0)$ & 0.1265 & 0.4309 & 0.7417 \\
\hline Concomitant pancreatitis, presence, $n(\%)$ & $4(9.3)$ & $2(12.5)$ & $2(8.0)$ & 0.5933 & 0.2701 & 0.0726 \\
\hline Family history of pancreatic cancer & $4(9.3)$ & $1(6.3)$ & $2(9.1)$ & 0.9335 & 0.9845 & 0.9827 \\
\hline \multicolumn{7}{|l|}{ Laboratory findings } \\
\hline Serum CEA, high ( $\geq 3.2 \mathrm{IU} / \mathrm{L})$ & $10(23.2)$ & $4(25.0)$ & $14(56.0)$ & 0.8737 & 0.0709 & 0.0928 \\
\hline Serum CA 19-9, high $(\geq 37$ IU/L) & $2(4.6)$ & $1(6.3)$ & $9(36.0)$ & 0.7282 & 0.0043 & 0.0957 \\
\hline Serum P-AMY, high $(\geq 50 \mathrm{IU} / \mathrm{L})$ & $11(25.5)$ & $2(12.5)$ & $6(24.0)$ & 0.1904 & 0.0495 & 0.6175 \\
\hline \multicolumn{7}{|l|}{ Imaging findings } \\
\hline Main pancreatic duct size, mean \pm SD & $6.51 \pm 3.90$ & $9.05 \pm 4.93$ & $8.36 \pm 6.19$ & 0.0900 & 0.7057 & 0.7815 \\
\hline Main pancreatic duct size, $(\geq 10 \mathrm{~mm}), n(\%)$ & $7(16.3)$ & $8(50.0)$ & $10(40.0)$ & 0.0456 & 0.2556 & 0.6649 \\
\hline Cystic size, mean $\pm \mathrm{SD}$ & $34.7 \pm 14.1$ & $38.1 \pm 28.9$ & $40.9 \pm 19.3$ & 0.9885 & 0.5333 & 0.7344 \\
\hline Enhancing mural nodule height $*,(\geq 5 \mathrm{~mm}), n(\%)$ & $12(27.9)$ & $8(50.0)$ & $29(84.0)$ & 0.2588 & $<0.0001$ & 0.0574 \\
\hline Cyst growth rate $\geq 5 \mathrm{~mm} / 2$ years, presence, $n(\%)$ & $3(7.0)$ & $0(0)$ & $2(8.0)$ & 0.5435 & 0.9892 & 0.5079 \\
\hline Abrupt change in caliber of pancreatic duct with distal pancreatic atrophy & $1(2.3)$ & $1(6.3)$ & $0(0)$ & 0.7593 & 0.7445 & 0.4531 \\
\hline Thickened/enhancing cyst walls, $n(\%)$ & $12(27.9)$ & $1(6.3)$ & $1(4.0)$ & 0.1842 & 0.0444 & 0.9558 \\
\hline Lymphadenopathy, $n(\%)$ & $1(2.3)$ & $1(6.3)$ & $0(0)$ & 0.7593 & 0.7445 & 0.4531 \\
\hline SUV-max, mean $\pm S D$ & $1.95 \pm 1.39$ & $3.17 \pm 2.82$ & $7.91 \pm 6.06$ & 0.4921 & $<0.0001$ & 0.0136 \\
\hline \multicolumn{7}{|l|}{ Other indicators } \\
\hline High-risk stigmata, positive, $n(\%)$ & $17(39.5)$ & $12(75.0)$ & $22(88.0)$ & 0.0442 & 0.0003 & 0.5482 \\
\hline Worrisome feature, positive, $n(\%)$ & $41(95.3)$ & $16(100.0)$ & $24(96.0)$ & 0.6762 & 0.9937 & 0.7336 \\
\hline
\end{tabular}

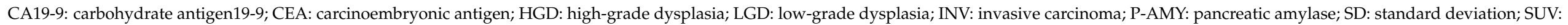
standardized uptake value. ${ }^{*}$ The mural nodule height was measured by contrast-enhanced computed tomography. 
A

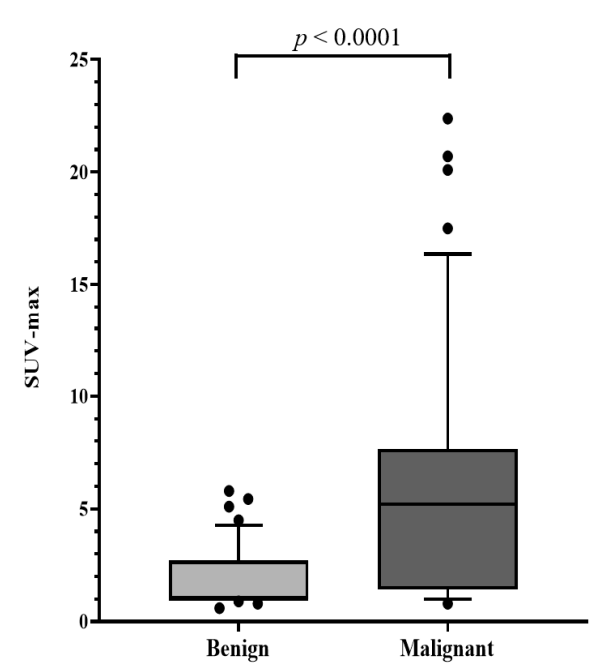

B

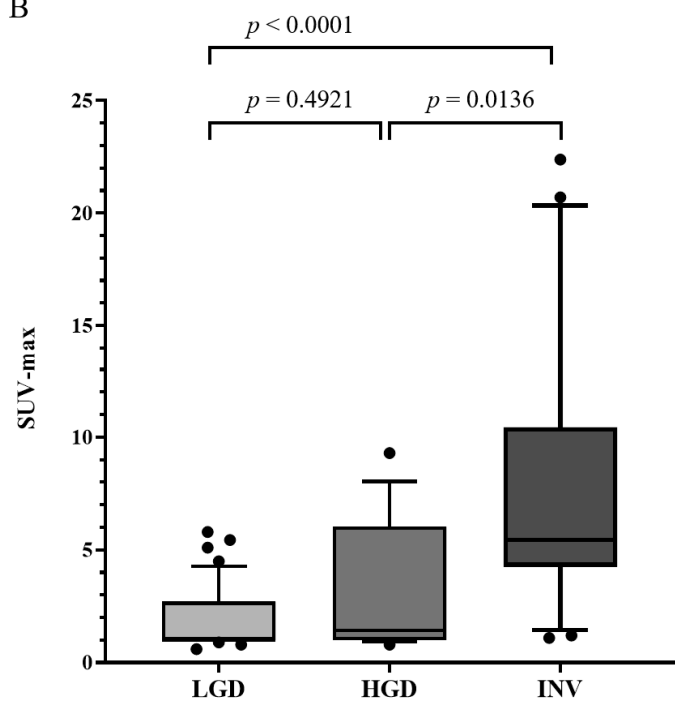

Figure 2. Box plot of the levels of SUV-max in the late phase. (A) Box plot of the levels of SUV-max in the benign group and malignant group are shown; SUV-max was significantly higher in the malignant group compared with the benign group $(p<0.0001)$. (B) Box plot of the levels of SUV-max in the LGD, HGD, and INV groups; SUV-max was significantly higher in the INV group that in the LGD and HGD groups ( $p<0.0001, p=0.0136$, respectively). There was no significant difference in the levels of SUV-max between the LGD and HGD groups. SUV-max: maximum standardized uptake value; LGD: low-grade dysplasia; HGD: high-grade dysplasia; INV: invasive cancer.

The ROC curve for comparison between malignant IPMN (HGD + INV) and LGD, with a cutoff score of 4.0, is shown in Figure 3A; the area under the curve (AUC) was 0.7714. Sensitivity and specificity were $90.7 \%$ and $61.0 \%$, respectively, when FDG uptake was used as a marker for benign/malignant differentiation of IPMNs. Among these 12 malignant IPMNs, IPMNs with HGD accounted for 9 cases (Table S1). From a different perspective, 9 out of 16 HGD patients (56.3\%) did not show FDG uptake. If the ROC curve was subtracted from the discriminability of FDG-PET/CT with INV only from LGD and HGD, the optimal cutoff score was 4.03 , AUC was 0.8634 , sensitivity was $80.0 \%$, and specificity was $86.2 \%$ (Figure 3B).

When the ROC curve was constructed using the mural nodule height measured by CT, MRI, and EUS for benign/malignant discrimination, the optimal cutoff values and AUC were as follows: CT $4.8 \mathrm{~mm}$ (AUC 0.762), MRI $5.4 \mathrm{~mm}$ (AUC 0.731), and EUS $9.9 \mathrm{~mm}$, (AUC 0.785).

3.3. Relationship between ${ }^{18}$ F-Fluorodeoxyglucose Uptake and Glucose Transporter 1 (GLUT-1) Expression in Immunohistochemistry for IPMNs

The selected samples comprised the five patients with the highest SUV-max values and the five patients with the lowest SUV-max values. The representative results of immunohistochemical staining for GLUT-1 are shown in Figure 4. In the LGD group, the incidence of GLUT-1-positive expression in the membrane was 20\% (2/10) (Figure 4 and Table S1). In contrast, GLUT-1 overexpression in the cytoplasm and basement membrane of cancer cells from IPMN with INV was detected (Figure 4). Among the 15 patients with HGD IPMNs, the incidence of GLUT-1-positive expression in the cell membrane was $40 \%$ $(6 / 15)$ (Table S1). GLUT-1 expression and the SUV-max corresponding to those of patients with IPMNs are summarized in Table S1, and more than half of patients with HGD IPMNs had lower GLUT-1 expression. 
$\mathbf{A}$

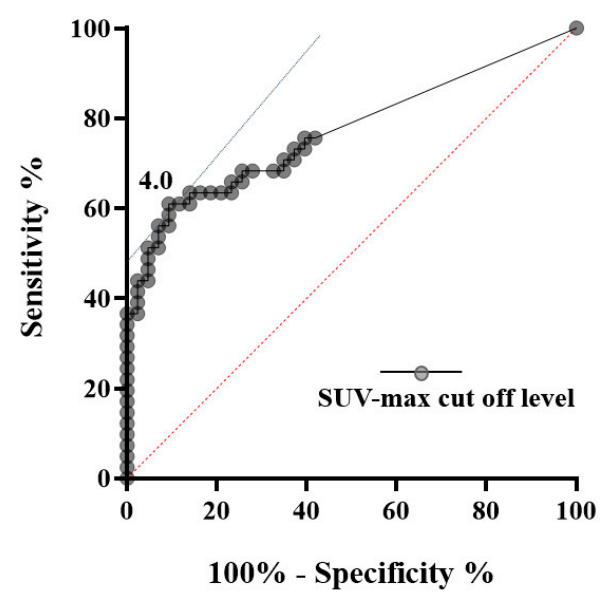

B

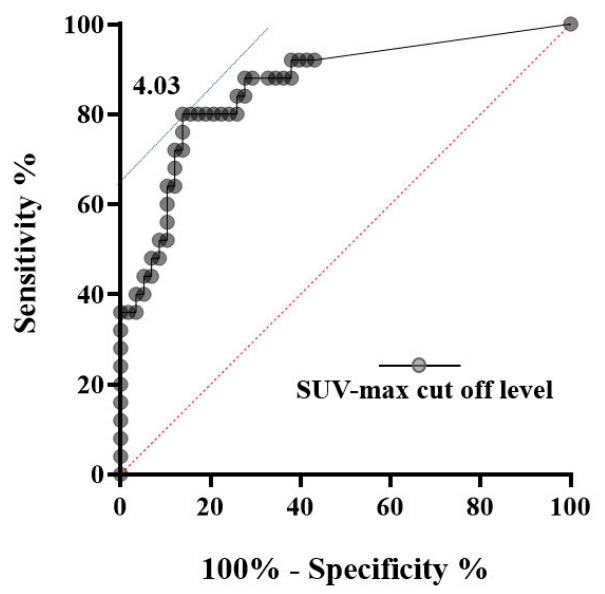

Figure 3. ROC curves. (A) Data on the curve represent SUV-max cutoff levels of 4.0 for the differential diagnosis of benign and malignant IPMNs according to the SUV-max of FDG-PET. (B) Data on the curve represent SUV-max cutoff levels of 4.03 for the differential diagnosis of low-grade dysplasia/high-grade dysplasia and invasive carcinoma according to the SUV-max of FDG-PET. ROC: receiver operating characteristic. SUV-max: maximum standardized uptake value; FDG-PET: 2- ${ }^{18} \mathrm{~F}$ fluoro-2-deoxy-D-glucose positron emission tomography.

\section{Representative images}

Low grade dysplasia No.6
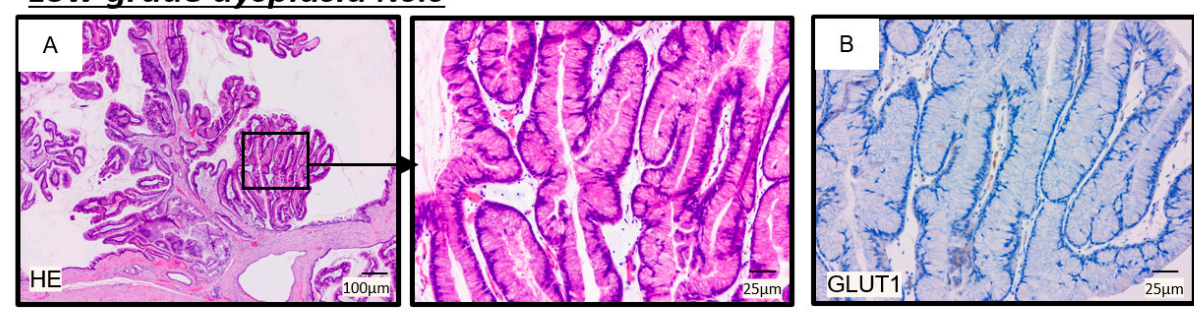

High grade dysplasia No.22
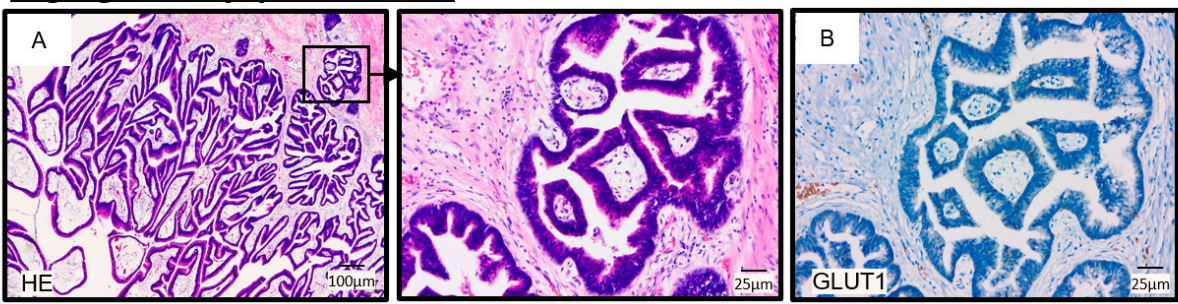

Invasive carcinoma No.30

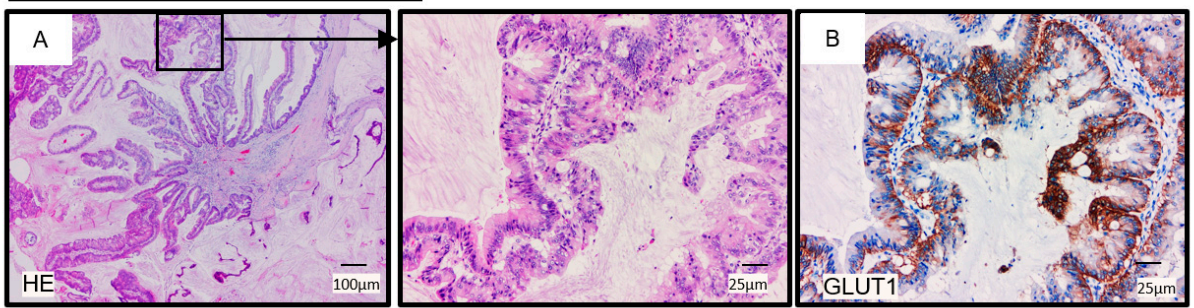

Figure 4. Representative hematoxylin and eosin (H\&E)-stained images and immunohistochemical images for GLUT1 in IPMNs with low-grade dysplasia, high-grade dysplasia, and invasive carcinoma are shown. (A) H\&E-stained sections of clinical specimens of IPMN. Areas in the boxed regions on the left are shown magnified on the right. (B) Immunohistochemical staining for GLUT-1. GLUT-1: glucose transporter 1; IPMN: intraductal papillary mucinous neoplasm. 


\subsection{Relationship between Mural Nodules and Preoperative Imaging Modality including} FDG-PET/CT

Pathologically proven mural nodules were revealed in 68 patients (80.9\%). Among them, PET uptake was positive in 44 . When the pathologically proven mural nodules and FDG uptake were confirmed one-on-one by two clinicians (Y.H. and O.H.), out of the 44 patients in whom FDG uptake was observed, FDG was accumulated in 41 of their mural nodules (93\%), excluding 3 in whom FDG was accumulated on the septum and cyst wall.

Additionally, there was a significant correlation between the size of the enhancing mural nodules measured by enhanced CT or enhanced EUS, and the pathological mural nodules $(\mathrm{R}=0.7943, p<0.001)$. Of the 33 patients with enhancing mural nodule height $<5 \mathrm{~mm}$ and negative FDG uptake, only 1 (3.0\%) INV was observed. Of the 23 patients with enhancing mural nodule height $\geq 10 \mathrm{~mm}$ and positive FDG uptake, $17(73.9 \%)$ INVs were observed.

Among FDG-uptake-positive IPMNs, weak significant correlations were shown between mural nodule height and SUV-max $(R=0.1474, p=0.0463)$ in all cases (Figure 5). However, when analyzed individually, there was no significant correlation among LGDs $(\mathrm{R}=0.2349, p=0.6190)$, HGDs $(\mathrm{R}=0.1113, p=0.3074)$, or INVs $(\mathrm{R}=-0.1901, p=0.8976)$.
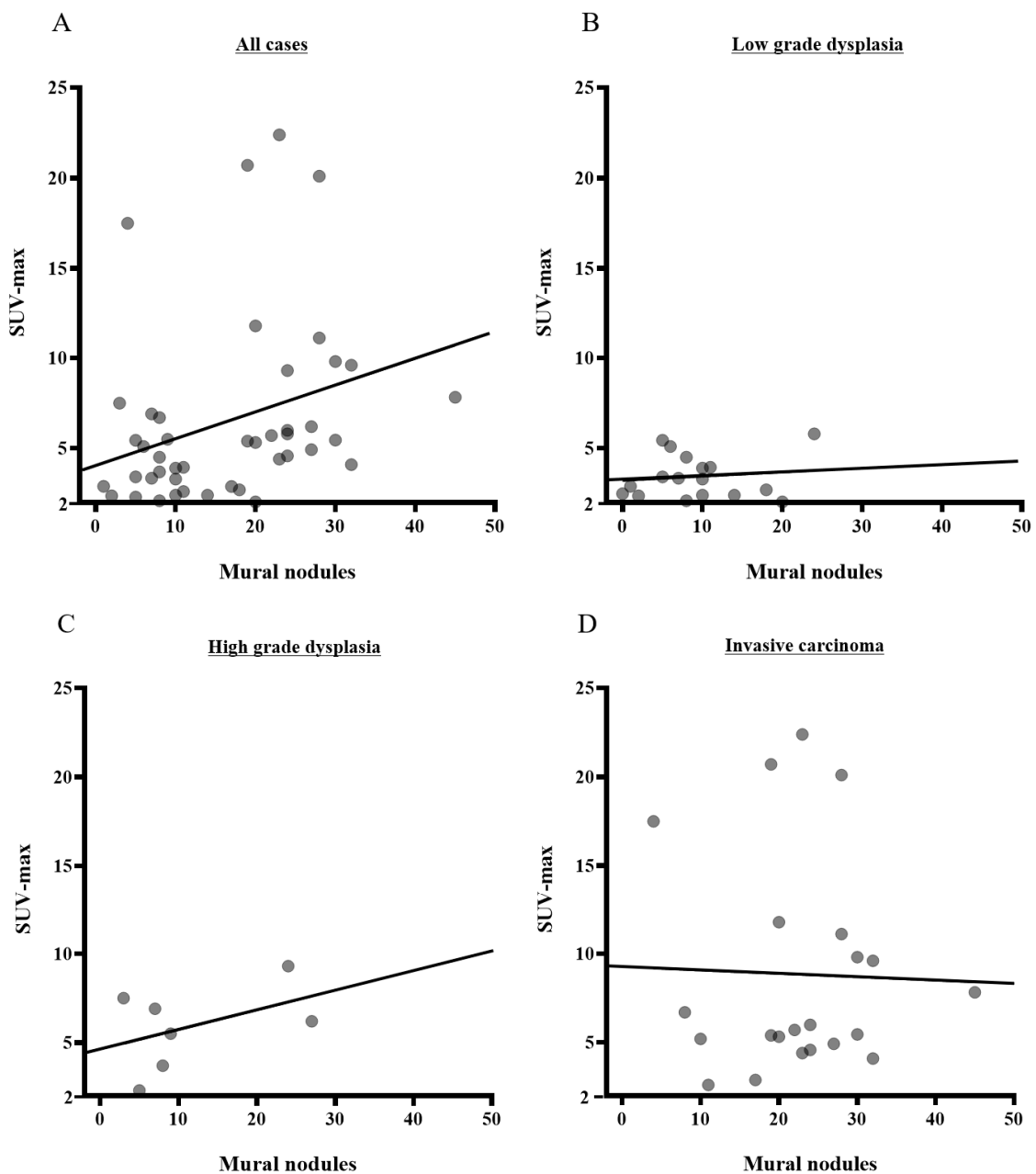

Figure 5. Correlations of the levels of SUV-max and mural nodule height by pathological grouping. (A) Significant correlations were found between mural nodule height and SUV-max in all IPMNs $(\mathrm{R}=0.1474, p=0.0463)$. (B) It was found that there was no significant correlation among LGDs $(\mathrm{R}=0.2349, p=0.6190)$. (C) It was found that there was no significant correlation among HGDs $(\mathrm{R}=0.1113, p=0.3074)$. (D) It was found that there was no significant correlation among INVs ( $\mathrm{R}=-0.1901, p=0.8976)$. SUV-max: maximum standardized uptake value; IPMN: intraductal papillary mucinous neoplasm. 


\section{Discussion}

As the definition of malignancy in IPMNs varies by country, region, and researcher, it has not yet been determined [12]. Most previous studies have included HGD and INV in one malignant category. Only a few studies have accurately assessed risk factors for each degree of dysplasia [27-30]. Thus, we focused on the diagnostic ability for the grade of dysplasia in IPMN using FDG-PET/CT. Based on what was revealed in the current study, we discuss the following three points in IPMNs: (1) differences in risk factors between HGD and INV; (2) FDG uptake and GLUT-1 expression in HGD and INV; and (3) the relationship between mural nodules and FDG in IPMNs.

First, treatment regimens for IPMNs involve surveillance protocol for LGD, minimally invasive surgery for LGD/HGD, and resection with lymph node dissection for INV. To facilitate surgical decision making for IPMNs, a highly accurate modality or finding that can distinguish the degree of malignancy in IPMN is required. Unfortunately, in our study, there were no useful factors that significantly separated the LGD, HGD, and INV groups from one another. However, enhancing mural nodule height and SUV-max were significantly higher in the INV group than in the LGD or HGD groups. Izumo et al. reported that the presence of an enhancing mural nodule height of $\geq 5 \mathrm{~mm}$, concomitant pancreatitis, and wall and septal thickening were significant risk factors for HGD [8]. In contrast, MPD size of $\geq 10 \mathrm{~mm}$, and an abrupt change in the caliber of the pancreatic duct with distal pancreatic atrophy, are risk factors for INV. However, contrary to the study by Izumo et al., our study showed that an MPD of $\geq 10 \mathrm{~mm}$ is a significant risk factor for HGD rather than INV. Moreover, enhancing mural nodules of $\geq 5 \mathrm{~mm}$ were also indicated as significant risk factors only for INV, but not for HGD. Multiple comparisons between the three groups (LGD, HGD, and INV) should be conducted, but Izumo et al. analyzed each of the two groups individually, which may have resulted in inaccurate statistical processing. These differences due to patient background may also have been affected [31]. From these conflicting results, no specific risk factors for HGD or INV could be determined. However, among the factors newly appearing during the follow-up of BD-IPMN, the appearance of mural nodules and MPD of $\geq 10 \mathrm{~mm}$ have been reported to be predictors of malignant IPMN [32]. Therefore, the appearance of any of these during the follow-up of BD-IPMN should warrant the consideration of the possibility of INV and surgical resection.

Second, regarding the diagnostic ability of FDG-PET/CT in IPMN, according to recent reviews, when the SUV-max cutoff was set between 1.3 and 3.0, FDG-PET/CT had malignant diagnostic ability, with a sensitivity of $80(62-100) \%$, a specificity of $95(71-100) \%$, and an accuracy of $87(76-97) \%[19,33]$. However, these reviews include both studies on INV alone and studies on INV and HGD. In a recent study involving 171 patients with IPMN [22], SUV-max was significantly higher in HGD and INV than in LGD IPMN, and the sensitivity and specificity of SUV-max to distinguish benign (LGD) from malignant (INV and HGD) disease were $87.2 \%$ and $80.0 \%$, respectively, when the cutoff value of SUV-max was set at 2.5. Moreover, several other reports analyzed the diagnostic ability of FDG-PET among LGD, HGD, and INV IPMNs; however, each report showed different results, and no significant conclusions could be obtained from the literature [23-25]. FDG is a glucose analog, and is moved into cells by glucose transporters, where it is then phosphorylated by hexokinases to FDG-6-phosphate [34]. In general, glucose-6-phosphatase activity in tumor cells is significantly reduced, and glucose transporters, which are involved in the intracellular transport of glucose, are often overexpressed. FDG-6-phosphate cannot be metabolized further in the glycolytic pathway, and stays in the cells [35]. Therefore, FDG uptake reflects glucose metabolism in the tissue, and activated cells enhance the uptake of FDG in many types of tumor cells relative to other normal cells [34]. Overexpression of GLUT-1 was reported to correlate with FDG uptake in various types of carcinoma (e.g., esophageal squamous-cell carcinoma, pancreatic carcinoma, and non-small-cell lung carcinoma) $[26,35,36]$. In the present study, the expression of GLUT-1 on immunohistochemical staining was almost nonexistent or weak among the HGD group, in which FDG uptake was low, whereas it was strong for the INV group, with its high FDG uptake. Hirashita et al. evaluated GLUT-1 and FDG-PET/CT using 39 IPMN 
pancreatic resection specimens, and found that the expression of GLUT-1 was significantly higher in carcinoma than in adenoma, showing that there is a correlation between SUV-max and the expression of GLUT-1 [37]. Oda et al. reported that HGD and INV showed higher expression of GLUT-1 than LGD [38]; their studies also found that there are tissue subtypes of IPMN, including many oncocytic types $(n=7)$ and the pancreatobiliary type $(n=21)$, which have higher expression of GLUT-1 than those seen in the gastric type $(n=30)$ and intensive type $(n=22)$. Our study did not include the oncocytic type, and the fact that only a few IPMNs were the pancreatobiliary type might have affected the difference in GLUT-1 expression for HGD. These results may indicate that HGD has oncologically low FDG uptake, and that FDG-PET/CT is unsuitable for HGD detection, although it is useful for differentiating between HGD and INV.

Third, how FDG accumulates at the site is unclear, as is its relationship with histopathological findings. Therefore, we investigated the relationship between FDG uptake and mural nodules, which are presumed to contain the most tumor cells. The height of enhancing mural nodules-presumed to contain tumor cells- has been considered a strong predictor of malignancy; however, even so, the PPV of HGD and INV is $\sim 60 \%$, and a malignant predictive diagnosis based on mural nodule height alone is not recommended [12,39-42]. In general, the problem is that SUVs have a partial volume effect when the lesion is small; thus, they may be underestimated [43]. In this study, IPMNs with HGD had a significantly lower mural nodule height than IPMNs with INV, which might be one of the reasons that FDG could not be detected. Previously, there was also no correlation found between mural nodules and FDG uptake [44]. Kawada et al. reported that they analyzed IPMNs with HGD or INV with a mural nodule of size $10 \mathrm{~mm}$ or more, finding that 23 out of 33 IPMNs $(69.6 \%)$ had focal lesions of HGD or INV in mural nodules or outside the nodule. These results may indicate that the size of the mural nodules is not correlated with the volume of tumor cells with the highest degree of dysplasia [45]. Since the tumor volume of HGD or INV is not directly reflected in the mural nodule height, this may be one of the reasons that the association between FDG uptake and mural nodule height is absent. FDG-PET/CT is presumed to reflect the tumor volume of neoplastic cells with active glucose metabolism; thus, FDG-PET/CT may have additional value that mural nodule height does not have. It can be suggested that if the mural nodule height is $5 \mathrm{~mm}$ or less and the SUV-max is 4 or less, it is highly unlikely that INV tumor cells are contained; conversely, if the SUV-max is 4 or more and the mural nodule height is $10 \mathrm{~mm}$ or more, there is a high possibility that the lesion is HGD or INV. However, it should be noted that SUV-max has a higher cutoff value in this study than that reported in other studies. Since SUV values are affected by the PET-scanner, image viewer, and the method of drawing the volume of interest, these factors might have affected our current results [19-21,23].

Our study has several limitations. First, SUV-max can also be affected by factors such as tumor size or patient height and weight. Consequently, the higher malignant cutoff value than that in the previous reports may be due to differences in these factors. Second, in the current study, FDG uptake of IPMNs was evaluated using contrast-enhanced CT, MRI, and EUS findings as a guide to determine the portion with SUV-max, because it was quite difficult to detect the mural nodule by PET/CT alone. Third, the number of IPMNs with INV and HGD was small, while that of IPMNs with LGD was high. Thus, there may be a risk of generalization in the results. However, the ratio of LGDs was $51.2 \%$ of the study population, which is comparable to that reported in previous studies $(32.2 \%-61.4 \%)[22,25,46,47]$. In addition, there was no clear difference in SUV-max between LGD and HGD patients ( $p=0.4921)$, and it was determined that a significant difference would not be obtained even if a larger research population was studied. Fourth, our study included patients who underwent pancreatectomy for IPMN, and had a selection bias because of the retrospective design. There were more patients in clinical practice without findings of suspicion of malignancy who were surveyed. Notably, data are only for patients with a high rate of malignancy in the population, and other risk factors for HGD or INV may not have been detected. Large-scale multicenter studies will reveal 
useful factors for differentiating LGD, HGD, and INV. The disadvantages of FDG-PET/CT are radiation exposure and high cost, and there was no clear difference in malignancy predictive ability compared to mural nodule height by CT, MRI, or EUS. However, FDG$\mathrm{PET} / \mathrm{CT}$ could determine whether the lesion might be invasive or non-invasive. IPMNs with INV may require more extensive pancreatectomy than non-invasive lesions and, therefore, our results offer critical information that may determine surgical treatment strategies. It may be necessary to elucidate new molecular oncological changes-which comprise differentially expressed genes and gene mutations that appear in the progression from LGD to HGD—and search for biomarkers.

\section{Conclusions}

FDG-PET/CT is useful in distinguishing between non-invasive and invasive IPMNs. Our results offer critical information that may determine surgical treatment strategies.

Supplementary Materials: The following are available online at https:/ /www.mdpi.com/article/10 .3390 / cancers13184633/s1, Table S1: Clinicopathological characteristics of patients who underwent immunohistochemical staining for GLUT-1 expression, Figure S1: Representative MRI, EUS, and PET/CT images for IPMNs with LGD, HGD, and INV.

Author Contributions: Conceptualization, Y.H. and H.K.; data curation, Y.H., K.T., M.J., H.N., and M.H.; formal analysis, Y.H. and T.I.; funding acquisition, T.O.; investigation, Y.H., H.O., Y.Y., Y.K., M.J., A.T. (Atsushi Tani), H.N., and M.H.; methodology, Y.M., Y.F., H.K., and T.O.; project administration, T.O.; supervision, T.O.; validation, H.M, M.N., A.N., T.Y., and A.T. (Akihide Tanimoto).; visualization, Y.H.; writing-original draft preparation, Y.H. and K.T.; writing-review and editing, H.K. and T.O. All authors have read and agreed to the published version of the manuscript.

Funding: This research was funded by JSPS KAKENHI, grant No. JP20H03753.

Institutional Review Board Statement: This study was approved by the Ethics Review Committee of Kagoshima University (approval no. 200277, date of approval: 19 March 2021).

Informed Consent Statement: Written informed consent was obtained from all enrolled patients.

Data Availability Statement: The data presented in this study are available on request from the corresponding author.

Acknowledgments: This work was supported by JSPS KAKENHI Grant Number JP20H03753.

Conflicts of Interest: The authors declare no conflict of interest.

\section{References}

1. Basturk, O.; Hong, S.M.; Wood, L.D.; Adsay, N.V.; Albores-Saavedra, J.; Biankin, A.V.; Brosens, L.A.; Fukushima, N.; Goggins, M.; Hruban, R.H.; et al. A Revised Classification System and Recommendations From the Baltimore Consensus Meeting for Neoplastic Precursor Lesions in the Pancreas. Am. J. Surg. Pathol. 2015, 39, 1730-1741. [CrossRef] [PubMed]

2. Hruban, R.H.; Takaori, K.; Klimstra, D.S.; Adsay, N.V.; Albores-Saavedra, J.; Biankin, A.V.; Biankin, S.A.; Compton, C.; Fukushima, N.; Furukawa, T.; et al. An illustrated consensus on the classification of pancreatic intraepithelial neoplasia and intraductal papillary mucinous neoplasms. Am. J. Surg. Pathol. 2004, 28, 977-987. [CrossRef]

3. Maitra, A.; Fukushima, N.; Takaori, K.; Hruban, R.H. Precursors to invasive pancreatic cancer. Adv. Anat. Pathol. 2005, 12, 81-91. [CrossRef] [PubMed]

4. Tanaka, M.; Chari, S.; Adsay, V.; Fernandez-del Castillo, C.; Falconi, M.; Shimizu, M.; Yamaguchi, K.; Yamao, K.; Matsuno, S. International consensus guidelines for management of intraductal papillary mucinous neoplasms and mucinous cystic neoplasms of the pancreas. Pancreatology 2006, 6, 17-32. [CrossRef] [PubMed]

5. Uehara, H.; Nakaizumi, A.; Ishikawa, O.; Iishi, H.; Tatsumi, K.; Takakura, R.; Ishida, T.; Takano, Y.; Tanaka, S.; Takenaka, A. Development of ductal carcinoma of the pancreas during follow-up of branch duct intraductal papillary mucinous neoplasm of the pancreas. Gut 2008, 57, 1561-1565. [CrossRef]

6. Maire, F.; Hammel, P.; Terris, B.; Paye, F.; Scoazec, J.Y.; Cellier, C.; Barthet, M.; O’Toole, D.; Rufat, P.; Partensky, C.; et al. Prognosis of malignant intraductal papillary mucinous tumours of the pancreas after surgical resection. Comparison with pancreatic ductal adenocarcinoma. Gut 2002, 51, 717-722. [CrossRef] [PubMed]

7. Yamada, S.; Fujii, T.; Hirakawa, A.; Takami, H.; Suenaga, M.; Hayashi, M.; Niwa, Y.; Hattori, N.; Iwata, N.; Kanda, M.; et al. Comparison of the Survival Outcomes of Pancreatic Cancer and Intraductal Papillary Mucinous Neoplasms. Pancreas 2018, 47, 974-979. [CrossRef] 
8. Izumo, W.; Higuchi, R.; Furukawa, T.; Yazawa, T.; Uemura, S.; Matsunaga, Y.; Shiihara, M.; Yamamoto, M. Comparison of patients with invasive intraductal papillary mucinous carcinoma and invasive ductal carcinoma of the pancreas: A pathological type- and stage-matched analysis. Scand. J. Gastroenterol. 2019, 54, 1412-1418. [CrossRef] [PubMed]

9. Cameron, J.L.; Riall, T.S.; Coleman, J.; Belcher, K.A. One thousand consecutive pancreaticoduodenectomies. Ann. Surg. 2006, 244, 10-15. [CrossRef]

10. Winter, J.M.; Cameron, J.L.; Campbell, K.A.; Arnold, M.A.; Chang, D.C.; Coleman, J.; Hodgin, M.B.; Sauter, P.K.; Hruban, R.H.; Riall, T.S.; et al. 1423 pancreaticoduodenectomies for pancreatic cancer: A single-institution experience. J. Gastrointest. Surg. 2006, 10, 1199-1210. [CrossRef] [PubMed]

11. Tanaka, M.; Fernández-del Castillo, C.; Adsay, V.; Chari, S.; Falconi, M.; Jang, J.Y.; Kimura, W.; Levy, P.; Pitman, M.B.; Schmidt, C.M.; et al. International consensus guidelines 2012 for the management of IPMN and MCN of the pancreas. Pancreatology 2012, 12, 183-197. [CrossRef]

12. Tanaka, M.; Fernández-Del Castillo, C.; Kamisawa, T.; Jang, J.Y.; Levy, P.; Ohtsuka, T.; Salvia, R.; Shimizu, Y.; Tada, M.; Wolfgang, C.L. Revisions of international consensus Fukuoka guidelines for the management of IPMN of the pancreas. Pancreatology 2017, 17, 738-753. [CrossRef]

13. Wilson, C.B. PET scanning in oncology. Eur. J. Cancer 1992, 28, 508-510. [CrossRef]

14. Pakzad, F.; Groves, A.M.; Ell, P.J. The role of positron emission tomography in the management of pancreatic cancer. Semin. Nucl. Med. 2006, 36, 248-256. [CrossRef]

15. Engledow, A.H.; Skipworth, J.R.; Pakzad, F.; Imber, C.; Ell, P.J.; Groves, A.M. The role of 18FDG PET/CT in the management of colorectal liver metastases. HPB 2012, 14, 20-25. [CrossRef]

16. Kitajima, K.; Kono, A.; Konishi, J.; Suenaga, Y.; Takahashi, S.; Sugimura, K. ${ }^{18}$ F-FDG-PET/CT findings of retroperitoneal tumors: A pictorial essay. Jpn. J. Radiol. 2013, 31, 301-309. [CrossRef]

17. Kurahara, H.; Maemura, K.; Mataki, Y.; Sakoda, M.; Iino, S.; Kawasaki, Y.; Arigami, T.; Mori, S.; Kijima, Y.; Ueno, S.; et al. Significance of (18)F-Fluorodeoxyglucose (FDG) Uptake in Response to Chemoradiotherapy for Pancreatic Cancer. Ann. Surg. Oncol. 2019, 26, 644-651. [CrossRef] [PubMed]

18. Kapoor, V.; McCook, B.M.; Torok, F.S. An introduction to PET-CT imaging. Radiographics 2004, 24, 523-543. [CrossRef] [PubMed]

19. Srinivasan, N.; Koh, Y.X.; Goh, B.K.P. Systematic review of the utility of 18-FDG PET in the preoperative evaluation of IPMNs and cystic lesions of the pancreas. Surgery 2019, 165, 929-937. [CrossRef] [PubMed]

20. Baiocchi, G.L.; Bertagna, F.; Gheza, F.; Grazioli, L.; Calanducci, D.; Giubbini, R.; Portolani, N.; Giulini, S.M. Searching for indicators of malignancy in pancreatic intraductal papillary mucinous neoplasms: The value of 18FDG-PET confirmed. Ann. Surg. Oncol. 2012, 19, 3574-3580. [CrossRef]

21. Pedrazzoli, S.; Sperti, C.; Pasquali, C.; Bissoli, S.; Chierichetti, F. Comparison of International Consensus Guidelines versus 18-FDG PET in detecting malignancy of intraductal papillary mucinous neoplasms of the pancreas. Ann. Surg. 2011, 254, 971-976. [CrossRef]

22. Hata, T.; Mizuma, M.; Motoi, F.; Ishida, M.; Morikawa, T.; Nakagawa, K.; Hayashi, H.; Kanno, A.; Masamune, A.; Kamei, T.; et al. An integrated analysis of host- and tumor-derived markers for predicting high-grade dysplasia and associated invasive carcinoma of intraductal papillary mucinous neoplasms of the pancreas. Surg. Today 2020, 50, 1039-1048. [CrossRef] [PubMed]

23. Ohta, K.; Tanada, M.; Sugawara, Y.; Teramoto, N.; Iguchi, H. Usefulness of positron emission tomography (PET)/contrastenhanced computed tomography (CE-CT) in discriminating between malignant and benign intraductal papillary mucinous neoplasms (IPMNs). Pancreatology 2017, 17, 911-919. [CrossRef]

24. Tomimaru, Y.; Takeda, Y.; Tatsumi, M.; Kim, T.; Kobayashi, S.; Marubashi, S.; Eguchi, H.; Tanemura, M.; Kitagawa, T.; Nagano, H.; et al. Utility of 2-[18F] fluoro-2-deoxy-D-glucose positron emission tomography in differential diagnosis of benign and malignant intraductal papillary-mucinous neoplasm of the pancreas. Oncol. Rep. 2010, 24, 613-620. [PubMed]

25. Saito, M.; Ishihara, T.; Tada, M.; Tsuyuguchi, T.; Mikata, R.; Sakai, Y.; Tawada, K.; Sugiyama, H.; Kurosawa, J.; Otsuka, M.; et al. Use of F-18 fluorodeoxyglucose positron emission tomography with dual-phase imaging to identify intraductal papillary mucinous neoplasm. Clin. Gastroenterol. Hepatol. 2013, 11, 181-186. [CrossRef]

26. Kita, Y.; Okumura, H.; Uchikado, Y.; Sasaki, K.; Omoto, I.; Matsumoto, M.; Setoyama, T.; Tanoue, K.; Mori, S.; Owaki, T.; et al. Clinical significance of ${ }^{18} \mathrm{~F}$-fluorodeoxyglucose positron emission tomography in superficial esophageal squamous cell carcinoma. Ann. Surg. Oncol. 2013, 20, 1646-1652. [CrossRef]

27. Izumo, W.; Higuchi, R.; Furukawa, T.; Yazawa, T.; Uemura, S.; Shiihara, M.; Yamamoto, M. Importance of each high-risk stigmata and worrisome features as a predictor of high-grade dysplasia in intraductal papillary mucinous neoplasms of the pancreas. Pancreatology 2020, 20, 895-901. [CrossRef] [PubMed]

28. Kobayashi, N.; Sugimori, K.; Shimamura, T.; Hosono, K.; Watanabe, S.; Kato, S.; Ueda, M.; Endo, I.; Inayama, Y.; Maeda, S.; et al. Endoscopic ultrasonographic findings predict the risk of carcinoma in branch duct intraductal papillary mucinous neoplasms of the pancreas. Pancreatology 2012, 12, 141-145. [CrossRef]

29. Ohtsuka, T.; Kono, H.; Nagayoshi, Y.; Mori, Y.; Tsutsumi, K.; Sadakari, Y.; Takahata, S.; Morimatsu, K.; Aishima, S.; Igarashi, H.; et al. An increase in the number of predictive factors augments the likelihood of malignancy in branch duct intraductal papillary mucinous neoplasm of the pancreas. Surgery 2012, 151, 76-83. [CrossRef] 
30. Correa-Gallego, C.; Do, R.; Lafemina, J.; Gonen, M.; D’Angelica, M.I.; DeMatteo, R.P.; Fong, Y.; Kingham, T.P.; Brennan, M.F.; Jarnagin, W.R.; et al. Predicting dysplasia and invasive carcinoma in intraductal papillary mucinous neoplasms of the pancreas: Development of a preoperative nomogram. Ann. Surg. Oncol. 2013, 20, 4348-4355. [CrossRef]

31. Jang, J.Y.; Park, T.; Lee, S.; Kim, Y.; Lee, S.Y.; Kim, S.W.; Kim, S.C.; Song, K.B.; Yamamoto, M.; Hatori, T.; et al. Proposed Nomogram Predicting the Individual Risk of Malignancy in the Patients With Branch Duct Type Intraductal Papillary Mucinous Neoplasms of the Pancreas. Ann. Surg. 2017, 266, 1062-1068. [CrossRef] [PubMed]

32. Oyama, H.; Tada, M.; Takagi, K.; Tateishi, K.; Hamada, T.; Nakai, Y.; Hakuta, R.; Ijichi, H.; Ishigaki, K.; Kanai, S.; et al. Longterm Risk of Malignancy in Branch-Duct Intraductal Papillary Mucinous Neoplasms. Gastroenterology 2020, 158, 226-237.e225. [CrossRef]

33. Serafini, S.; Sperti, C.; Brazzale, A.R.; Cecchin, D.; Zucchetta, P.; Pierobon, E.S.; Ponzoni, A.; Valmasoni, M.; Moletta, L. The Role of Positron Emission Tomography in Clinical Management of Intraductal Papillary Mucinous Neoplasms of the Pancreas. Cancers 2020, 12, 807. [CrossRef] [PubMed]

34. Leung, K. [(18)F]Fluoro-2-deoxy-2-D-glucose. In Molecular Imaging and Contrast Agent Database (MICAD); National Center for Biotechnology Information (US): Bethesda, MD, USA, 2004.

35. Kaida, H.; Azuma, K.; Kawahara, A.; Yasunaga, M.; Kitasato, Y.; Hattori, S.; Taira, T.; Ureshino, H.; Kage, M.; Ishii, K.; et al. The correlation between FDG uptake and biological molecular markers in pancreatic cancer patients. Eur. J. Radiol. 2016, 85, 1804-1810. [CrossRef] [PubMed]

36. Choi, W.H.; Yoo, I.R.; Kim, T.J.; Lee, K.Y.; Kim, Y.K. Is the Glut expression related to FDG uptake in PET/CT of non-small cell lung cancer patients? Technol. Health Care. 2015, 23 (Suppl. 2), S311-S318. [CrossRef]

37. Hirashita, T.; Hirashita, Y.; Iwashita, Y.; Endo, Y.; Kiyonaga, M.; Matsumoto, S.; Hijiya, N.; Moriyama, M.; Murakami, K.; Inomata, M. S6 ribosomal protein phosphorylation is associated with malignancy of intraductal papillary mucinous neoplasm of the pancreas. Ann. Gastroenterol. Surg. 2020, 4, 571-579. [CrossRef]

38. Oda, Y.; Aishima, S.; Shindo, K.; Fujino, M.; Mizuuchi, Y.; Hattori, M.; Miyazaki, T.; Tanaka, M.; Oda, Y. SLC2A1/GLUT1 expression in mural nodules of intraductal papillary mucinous neoplasm of the pancreas. Hum. Pathol. 2017, 65, 71-78. [CrossRef]

39. Schmidt, C.M.; White, P.B.; Waters, J.A.; Yiannoutsos, C.T.; Cummings, O.W.; Baker, M.; Howard, T.J.; Zyromski, N.J.; Nakeeb, A.; DeWitt, J.M.; et al. Intraductal papillary mucinous neoplasms: Predictors of malignant and invasive pathology. Ann. Surg. 2007, 246, 644-651. [CrossRef]

40. Uehara, H.; Ishikawa, O.; Katayama, K.; Kawada, N.; Ikezawa, K.; Fukutake, N.; Takakura, R.; Takano, Y.; Tanaka, S.; Takenaka, A. Size of mural nodule as an indicator of surgery for branch duct intraductal papillary mucinous neoplasm of the pancreas during follow-up. J. Gastroenterol. 2011, 46, 657-663. [CrossRef]

41. Akita, H.; Takeda, Y.; Hoshino, H.; Wada, H.; Kobayashi, S.; Marubashi, S.; Eguchi, H.; Tanemura, M.; Mori, M.; Doki, Y.; et al. Mural nodule in branch duct-type intraductal papillary mucinous neoplasms of the pancreas is a marker of malignant transformation and indication for surgery. Am. J. Surg. 2011, 202, 214-219. [CrossRef]

42. Nguyen, A.H.; Toste, P.A.; Farrell, J.J.; Clerkin, B.M.; Williams, J.; Muthusamy, V.R.; Watson, R.R.; Tomlinson, J.S.; Hines, O.J.; Reber, H.A.; et al. Current recommendations for surveillance and surgery of intraductal papillary mucinous neoplasms may overlook some patients with cancer. J. Gastrointest. Surg. 2015, 19, 258-265. [CrossRef]

43. Soret, M.; Bacharach, S.L.; Buvat, I. Partial-volume effect in PET tumor imaging. J. Nucl. Med. 2007, 48, 932-945. [CrossRef]

44. Takanami, K.; Hiraide, T.; Tsuda, M.; Nakamura, Y.; Kaneta, T.; Takase, K.; Fukuda, H.; Takahashi, S. Additional value of FDG $\mathrm{PET} / \mathrm{CT}$ to contrast-enhanced CT in the differentiation between benign and malignant intraductal papillary mucinous neoplasms of the pancreas with mural nodules. Ann. Nucl. Med. 2011, 25, 501-510. [CrossRef]

45. Kawada, N.; Uehara, H.; Nagata, S.; Tsuchishima, M.; Tsutsumi, M.; Tomita, Y. Mural nodule of 10 mm or larger as predictor of malignancy for intraductal papillary mucinous neoplasm of the pancreas: Pathological and radiological evaluations. Pancreatology 2016, 16, 441-448. [CrossRef] [PubMed]

46. Shimizu, Y.; Hijioka, S.; Hirono, S.; Kin, T.; Ohtsuka, T.; Kanno, A.; Koshita, S.; Hanada, K.; Kitano, M.; Inoue, H.; et al. New Model for Predicting Malignancy in Patients With Intraductal Papillary Mucinous Neoplasm. Ann. Surg. 2020, 272, 155-162. [CrossRef] [PubMed]

47. Iwaya, H.; Hijioka, S.; Mizuno, N.; Kuwahara, T.; Okuno, N.; Tajika, M.; Tanaka, T.; Ishihara, M.; Hirayama, Y.; Onishi, S.; et al. Usefulness of septal thickness measurement on endoscopic ultrasound as a predictor of malignancy of branched-duct and mixed-type intraductal papillary mucinous neoplasm of the pancreas. Dig. Endosc. 2019, 31, 672-681. [CrossRef] [PubMed] 